\title{
Low-Complexity Suppression of Adjacent Channel Interference in FDD Transceiver
}

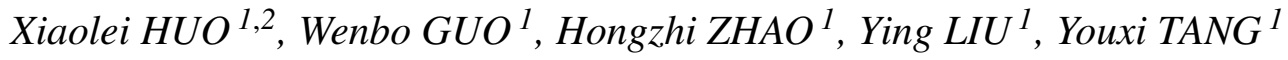 \\ ${ }^{1}$ National Key Laboratory of Science and Technology on Communications, University of Electronic Science and \\ Technology of China, 611731, Chengdu, China \\ 2 Department of Electronic and Optical Engineering, Shijiazhuang Campus of Army Engineering University, 050003, \\ Shijiazhuang, China
}

liuying850613@uestc.edu.cn

Submitted April 14, 2021 / Accepted October 13, 2021

\begin{abstract}
The transmitter-induced adjacent channel interference (ACI) due to power amplifier nonlinearity poses severe desensitization to the receiver in frequency-division $d u$ plexing transceivers. To tackle this issue, this paper proposed a digital suppression method with a low-complexity circuit structure to eliminate the interference. The transmitter baseband signal and leakage were employed to estimate the system nonlinear parameters in the digital baseband domain, and the interference was regenerated and then subtracted from the received signal. The proposed method can simplify the circuit structure and facilitate engineering implementation in practice. The simulation and experimental results show that the proposed method can suppress about $25 \mathrm{~dB} A C I$, which can effectively improve the signal-to-interference-plus-noise ratio of the desired signal.
\end{abstract}

\section{Keywords}

ACI suppression, parameters estimation, signal regeneration, nonlinear distortion, low-complexity

\section{Introduction}

Wireless communication system usually achieves frequency-division duplex (FDD) operation by using duplexer. In these FDD applications, it is still a big challenge to provide isolation simultaneously over both the TX and RX branches [1].

To eliminate the interference, bandpass filtering is usually conducted to isolate the TX and RX branches in duplexer [2], [3]. However, the steep cut-off characteristic of the filter will result in a larger duplexer and higher cost. In the case of narrow separation, the duplexer filter may fail to block the interference induced by the TX branch.

In order to obtain a smaller and cheaper duplexer, people have to sacrifice the isolation bandwidth of the duplexer. As a result, traditional duplexers can provide $45-55 \mathrm{~dB}$ isolation on the transmit branch but only achieve $20-30 \mathrm{~dB}$ isolation on the receiving branch [4]. A direct consequence of the insufficient isolation bandwidth is that the transmitter leakage through the duplexer will pose serious interference to the desired signal [5], [6]. Therefore, the suppression and elimination of the transmitter leakage have become a hot issue of recent research [7], [8].

Regenerating and eliminating the leakage at the receiver has been considered as an effective technique to suppress interference. In [9-11], the radio frequency (RF) signal generated by the auxiliary branch was subtracted from the received signal to suppress the interference and finally achieved $21.6 \mathrm{~dB}$ interference suppression. However, the isolation bandwidth is limited by frequency-domain variation in the antenna and balancing reflection coefficients in these methods. Therefore, providing isolation in both the bands entails increased RF complexity in the balancing network. Higherorder RF processing not only increases the size and cost of the radio frequency front end but also requires higher-order multi-dimensional optimization to tune the circuit parameters [12], [13].

As shown in [14], [15], a low sampling rate digital baseband suppression model was proposed to eliminate the transmitter leakage, and eventually, the leakage is suppressed by approximately $20 \mathrm{~dB}$. However, the focus of the above research is to reduce the aliasing effect on interference suppression caused by the sampling rate reduction. The sampling rate is reduced from the traditional 368.64 MSPS to 61.44 MSPS for a $20 \mathrm{MHz}$ baseband signal in [14]. Although the sampling rate has been greatly reduced, the analog-to-digital converter (ADC) sampling bandwidth is three times the baseband signal bandwidth and still requires a certain amount of hardware resources.

To tackle this issue, this paper proposed a memory polynomial (MP) method with duplexer fitting, which employed the transmitter leakage and baseband signal to estimate the system nonlinear parameters and then regenerated and eliminated the interference. Compared with the RF domain suppression method, the proposed method effectively simplifies 
the circuit structure and is easy to implement in engineering. Since the ADC output signal can be directly used for parameter estimation, the required ADC sampling bandwidth is the same as the bandwidth of the baseband signal, which also reduces the requirements for hardware resources.

The remainder of this paper is organized as follows. Detailed system architecture and principle of operation are presented in Sec. 2. The simulation results are given in Sec. 3, and the experimental results are reported in Sec. 4. Finally, Section 5 concludes this paper.

\section{Adjacent Channel Interference Suppression}

\subsection{System Model Block Diagram}

The detailed system block diagram of the proposed method is illustrated in Fig. 1. The transmitter and receiver share a common antenna through a duplexer. The TX frequency is $\omega_{2}$, the RX frequency is $\omega_{1}$, and we may assume that $\Delta \omega=\omega_{1}-\omega_{2}$. The baseband signal of the transmitter passes through a digital-to-analog converter (DAC) and an upconversion circuit, and the RF signal after power amplification enters the duplexer. Due to the PA nonlinear characteristics, the RF signal spectrum will broaden, then the extended sidelobe will leak into the receiver through the duplexer, causing adjacent channel interference (ACI) to the desired signal.

To mitigate the ACI, an auxiliary branch was added in the transceivers to estimate the system nonlinear characteristics. The transmitter baseband signal $x[n]$ and the receiver ADC output signal $r[n]$ were employed to regenerate the interference. Finally, the regenerated interference was subtracted from the received signal to suppress the ACI.

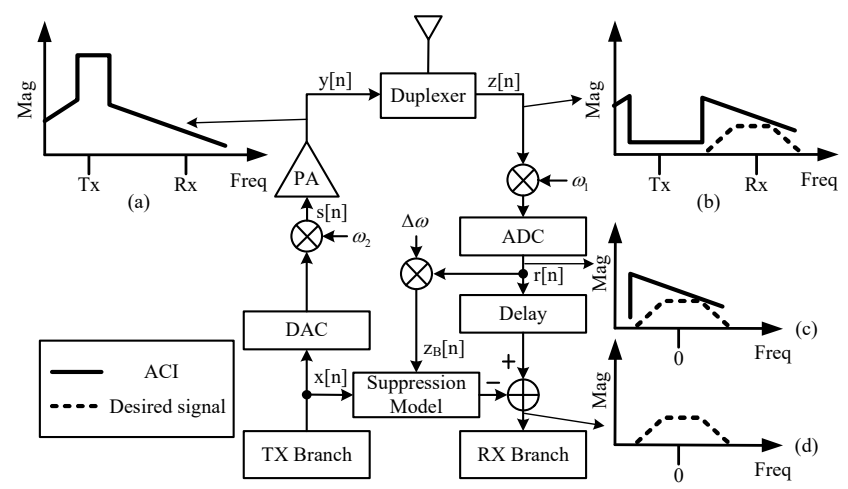

Fig. 1. The system block diagram. (a) Due to the nonlinearity of the power amplifier, the spectrum is broadened by the intermodulation products of the TX signal. (b) Due to the insufficient isolation of the duplex filter, the filtered spectrum regrowth will still seriously interfere with the desired signal. (c) The ACI and the desired signal are downconverted to baseband signals. (d) After subtracting the reconstructed signal to suppress the ACI, the SNR of desired signal is improved.
The power amplifier (PA) in the transmitter is the main reason for the introduction of system nonlinear distortion. In order to accurately regenerate the ACI, this paper used memory polynomial (MP) with duplexer fitting to describe the nonlinear characteristics of the PA [16].

The input signal $s[n]$ of the PA can be denoted as

$$
s[n]=x[n] \mathrm{e}^{\mathrm{j} \omega_{2} n}
$$

where $x[n]$ is the baseband signal of the transmitter, $\omega_{2}$ is the TX frequency.

The output signal $y[n]$ of the PA can be obtained as [17]

$$
y[n]=\sum_{k=0}^{K} \sum_{q=0}^{Q-1} w_{k q} s[n-q]|s[n-q]|^{2 k+1}
$$

where $Q$ is the maximum memory depth of the memory polynomial, $(2 K+1)$ is the maximum nonlinear order, $w_{k q}$ is the complex coefficient of the MP model.

Substituting (1) into (2), it yields

$$
\begin{aligned}
y[n] & =\sum_{k=0}^{K} \sum_{q=0}^{Q-1} w_{k q} x[n-q] \mathrm{e}^{\mathrm{j} \omega_{2}(n-q)}\left|x[n-q] \mathrm{e}^{\mathrm{j} \omega_{2}(n-q)}\right|^{2 k+1} \\
& =\sum_{k=0}^{K} \sum_{q=0}^{Q-1} c_{k q} x[n-q]|x[n-q]|^{2 k+1} \mathrm{e}^{\mathrm{j} \omega_{2} n},
\end{aligned}
$$

where $c_{k q}=\exp \left(-\mathrm{j} \omega_{2} q\right) w_{k q}$ denotes the equivalent complex coefficients of the MP model [17].

The input signal $z[n]$ of the receiver can be denoted as

$$
z[n]=y[n] * h[n]+(e[n]+w[n]) \mathrm{e}^{\mathrm{j} \omega_{1} n}
$$

where $h[n]$ is the impulse response of the duplexer, the symbol $(*)$ denotes the convolution operation, $e[n]$ is the desired signal, and $w[n]$ is the gaussian white noise.

Then the baseband signal $r[n]$ can be obtained as

$$
\begin{aligned}
r[n]= & \left(\sum_{k=0}^{K} \sum_{q=0}^{Q-1} c_{k q} x[n-q]|x[n-q]|^{2 k+1} * h_{\mathrm{B}}[n]\right) \mathrm{e}^{-\mathrm{j} \Delta \omega n} \\
& +e[n]+w[n],
\end{aligned}
$$

where $h_{\mathrm{B}}[n]$ is the equivalent baseband representation of $h[n]$.

In order to facilitate the problem analysis, Figure 2 shows the spectra of the baseband signal $x[n], z_{\mathrm{B}}[n]$ (the equivalent baseband representation of the transmitted signal $y[n]$ ) and $y_{\mathrm{B}}[n]$ (the equivalent baseband representation of the ACI $z[n])$. It can be easily seen from the figure that the maximum sidelobe of the ACI is approximately $-20 \mathrm{dBm}$, which is much larger than the desired signal. Therefore, the leaked ACI must be suppressed to improve the signal-tointerference-plus-noise ratio (SINR) of the desired signal. 


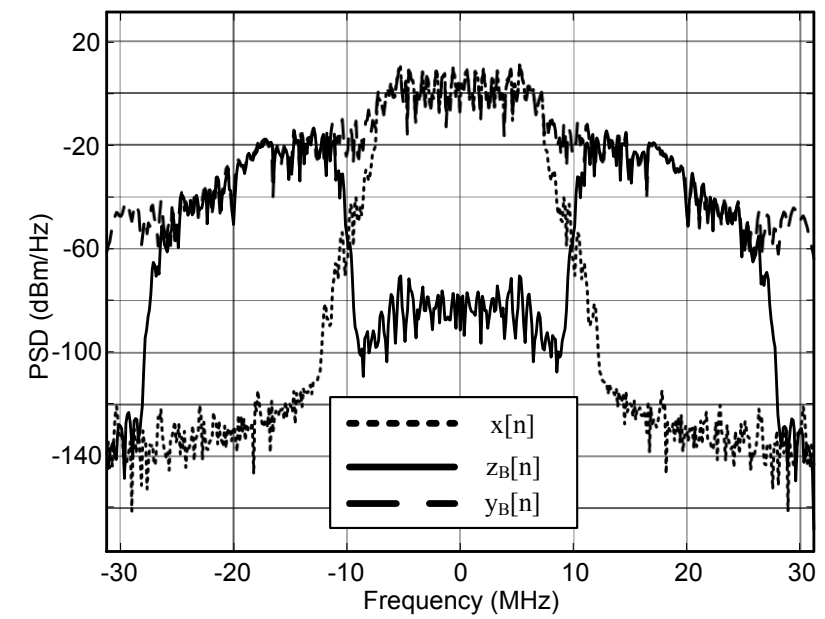

Fig. 2. The spectra of $x[n], z_{\mathrm{B}}[n]$ and $y_{\mathrm{B}}[n]$.

\subsection{Principle of ACI Suppression}

Before starting ACI suppression, the system convergence solution must be obtained through parameters training, i.e., when the receiver does not contain the desired signal, the training sequence is used to obtain the system nonlinear parameters. In the subsequent ACI suppression process, the obtained parameters and the baseband signal $x[n]$ were used to regenerate and eliminate the ACI, thereby improving the SINR of the desired signal.

From the above analysis, it is not difficult to deduce that the estimation of the parameters in the training phase is crucial for interference suppression.

The system nonlinear parameters are calculated by the suppression model in the auxiliary branch. The detailed block diagram of the model is shown in Fig. 3, where DPF stands for duplexer fitting. The signal processing that needs to be completed in the suppression model mainly includes

\section{Upconversion}

In the receiver, the ADC output baseband signal $r[n]$ is re-upconverted to obtain $z_{\mathrm{B}}[n]$, i.e., the nonlinear out-ofband components of the transmitted signal $y[n]$.

$$
\begin{aligned}
& z_{\mathrm{B}}[n]= r[n] \mathrm{e}^{\mathrm{j} \Delta \omega n} \\
&=\left(\sum_{k=0}^{K} \sum_{q=0}^{Q-1} c_{k q} x[n-q]|x[n-q]|^{2 k+1}\right) * h_{\mathrm{B}}[n] \\
&+w[n] \mathrm{e}^{\mathrm{j} \Delta \omega n} .
\end{aligned}
$$

\section{Nonlinear Parameters Estimation}

Based on the baseband signal $x[n]$ and transmitted signal $y[n]$, the system nonlinear parameters can be obtained through MP or GMP method [16], [17]. For the convenience of representation, (5) is rewritten into matrix form

$$
\mathbf{r}=\mathbf{X} \mathbf{\Omega}+\mathbf{w}
$$

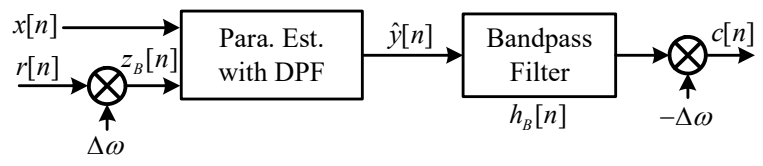

Fig. 3. The detailed block diagram of suppression model.

where $\mathbf{X}$ denotes $N \times k p$ matrices of the baseband signal $x[n]$, $N$ is the length of the observed signal, $k$ is the polynomial order, $p$ is the memory depth.

$$
\mathbf{X}=\left[\mathbf{X}_{0}, \mathbf{X}_{1}, \ldots, \mathbf{X}_{N-1}\right]^{\mathrm{T}}
$$

where $\mathbf{X}_{n}=\left[x_{n, 0,0}, \ldots, x_{n, k, 0}, \ldots, x_{n, 0, p}, \ldots, x_{n, k, p}\right]^{\mathrm{T}}$, $x_{n, k, p}=x_{n}(k, p)=x[n-p]|x[n-p]|^{(2 k+1)}$, and $\mathbf{\Omega}$ is $k p \times 1$ polynomial parameters need to be estimated.

\section{Duplexer Fitting}

After filtering by the duplexer, we can obtain $z[n]$ from the transmitted signal $y[n]$. For the convenience of presentation, the process is also rewritten into matrix form

$$
\widetilde{\mathbf{r}}=\mathbf{H r}=\mathbf{H X \Omega}
$$

where $\widetilde{\mathbf{r}}$ denotes the observated signal with noise, H denotes $\left(N+N_{\mathrm{F}}-1\right) \times N$ matrices constructed by the impulse response coefficients of duplexer $h_{\mathrm{B}}[n]=$ $h_{\mathrm{B}}[0], h_{\mathrm{B}}[1], \ldots, h_{\mathrm{B}}[N-1]$,

$$
\mathbf{H}=\left[\begin{array}{cccc}
h_{\mathrm{B}}[0] & 0 & \ldots & 0 \\
h_{\mathrm{B}}[1] & h_{\mathrm{B}}[0] & \ldots & 0 \\
\vdots & \vdots & \vdots & \vdots \\
h_{\mathrm{B}}[M-1] & h_{\mathrm{B}}[M-2] & \ldots & h_{\mathrm{B}}[0] \\
\vdots & \vdots & \vdots & \vdots \\
h_{\mathrm{B}}[N-1] & h_{\mathrm{B}}[N-2] & \ldots & h_{\mathrm{B}}[N-M] \\
\vdots & \vdots & \vdots & \vdots \\
0 & 0 & \ldots & h_{\mathrm{B}}[N-1]
\end{array}\right]
$$

However, it should be noted in (9) that the observated signal $\widetilde{\mathbf{r}}$ length is $N$, the right matrices length is $N+N_{\mathrm{F}}-1$, which makes it impossible to directly solve the equation. The reason is that in band-pass filtering, when the data of the current frame begins to enter the shift register, the data of the previous frame has not been completely moved out, resulting in cross-coupling between adjacent frames. Therefore, the left side of the (9) can process continuous signals, while the right side of the equation can only process finite-length signals according to the matrix multiplication rules, resulting in different signal lengths on both sides of the equation.

Therefore, it is necessary to process (9) as

$$
\Xi \widetilde{\mathbf{r}}=\Xi \mathbf{H X \Omega}
$$

where $\Xi$ denotes signal truncation. The signal length on both sides of the equation is equal by preserving the data in the middle of the frame. In order to completely eliminate the data with cross-coupling errors at the beginning or end of the 
frame, the length of the truncated frame should be no greater than $N-2 N_{\mathrm{F}}$

Then the formula can be easily solved by Least Squares method [18]

$$
\widehat{\mathbf{\Omega}}=\left((\boldsymbol{\Xi} \mathbf{H X})^{\mathrm{H}}(\Xi \mathbf{H X})\right)^{-1}(\Xi \mathbf{H X})^{\mathrm{H}}(\Xi \widetilde{\mathbf{r}})
$$

where $(\boldsymbol{\Xi H X})^{\mathrm{H}}$ denotes the conjugate transpose matrix of $\Xi \mathbf{H X},(\Xi \mathbf{H X})^{-1}$ denotes the inverse matrix of $\boldsymbol{\Xi} \mathbf{H X}$.

When the iterative calculation converged, the estimated parameters $\widehat{\boldsymbol{\Omega}}$ and the baseband signal $x[n]$ can be used to reconstruct the transmitted signal $\widehat{y}[n]$

$$
\widehat{y}[n]=\mathbf{X} \widehat{\mathbf{\Omega}}=\sum_{k=0}^{K} \sum_{q=0}^{Q-1} \widehat{c}_{k q} x[n-q]|x[n-q]|^{2 k+1} .
$$

\section{Filtering and Downconversion}

To suppress the ACI, it is necessary to filter the regenerated signal $\widehat{y}[n]$ again to take out the out-of-band nonlinear components, and then downconverted the filtered signal to obtain the zero-frequency baseband signal $c[n]$

$$
c[n]=\left(\sum_{k=0}^{K} \sum_{q=0}^{Q-1} \widehat{c}_{k q} x[n-q]|x[n-q]|^{2 k+1} * h_{\mathrm{B}}[n]\right) \mathrm{e}^{-\mathrm{j} \Delta \omega n} .
$$

\section{ACI Suppression}

By fine-tuning the time delay, the waveform of the received signal $r[n]$ is aligned with the regenerated signal $c[n]$ in the time domain [19]. Then $r[n]$ is subtracted from the received signal to suppress the ACI. The residual interference after suppression is given as

$$
\begin{aligned}
r[n]-c[n] & =\left(\sum_{k=0}^{K} \sum_{q=0}^{Q-1}\left(c_{k q}-\widehat{c}_{k q}\right) x[n-q]|x[n-q]|^{2 k+1}\right. \\
& \left.* h_{\mathrm{B}}[n]\right) \mathrm{e}^{-\mathrm{j} \Delta \omega n}+w[n] .
\end{aligned}
$$

It can be easily seen that the residual interference is proportional to the estimation error of the MP coefficients $c_{k q}-\widehat{c}_{k q}$.

From the above discussion, it is not difficult to see that the overall system complexity is considerably lower, and the power consumption of the system can be dramatically reduced because a much lower clock rate is required.

\section{Simulation Results}

To verify the feasibility of the proposed method, computer simulation was conducted in Matlab in this section. Table 1 shows the settings of various parameters in the simulation system, in which a finite impulse response band-pass filter is used to approximate the filtering characteristics of the duplexer.

\begin{tabular}{|c|c|}
\hline System parameter & Value \\
\hline Signal bandwidth & $20 \mathrm{MHz}$ \\
\hline TX frequency & $2.412 \mathrm{GHz}$ \\
\hline RX frequency & $2.437 \mathrm{GHz}$ \\
\hline Stop frequency 1 / Pass frequency 1 & $9 / 13 \mathrm{MHz}$ \\
\hline Stop frequency 2 / Pass frequency 2 & $27 / 31 \mathrm{MHz}$ \\
\hline Band attenuation & $50 \mathrm{~dB}$ \\
\hline
\end{tabular}

Tab. 1. Parameter settings of simulated system.

Since more and more communication systems are working in the license-free $2.4 \mathrm{GHz}$ industrial scientific medical (ISM) frequency bands, where neither resource planning nor bandwidth allocation can be guaranteed, so ACI in the ISM frequency band is also the most severe. To demonstrate the ACI suppression effect of the proposed method, we use $2.4 \mathrm{GHz}$ as the research object in the following simulation and experiment.

The transmitter baseband signal enters the duplexer after passing through the power amplifier with memory effect. The complex coefficients of the MP model of PA are set as follows [16]

$$
\begin{aligned}
& w_{10}=1.0513+0.0904 \mathrm{j}, \quad w_{30}=-0.0542-0.2900 \mathrm{j}, \\
& w_{50}=-0.9657-0.7028 \mathrm{j}, w_{11}=-0.0680-0.0023 \mathrm{j}, \\
& w_{31}=0.2234+0.2317 \mathrm{j}, \quad w_{51}=-0.2451-0.3735 \mathrm{j}, \\
& w_{12}=0.0289-0.0054 \mathrm{j}, \quad w_{32}=-0.0621-0.0932 \mathrm{j}, \\
& w_{52}=0.1229+0.1508 \mathrm{j} .
\end{aligned}
$$

The transmitter baseband signal and the upconverted ACI are simultaneously sent to the auxiliary branch for parameters estimation. To have a better observation of the estimation accuracy, more tests of ACI suppression with different system configurations were performed. Residual interference after suppression was evaluated against different nonlinear orders and memory depths [20].

The interference cancellation process is actually performed in the baseband. In order to facilitate the observation of the corresponding relationship between the interference and the transmitted signal, in the following experimental results, we will give the equivalent baseband spectra of the receiver and the transmitter signals side by side on the frequency axis.

Under the conditions of $K=3$ and $Q=3$, the corresponding ACI suppression results are shown in Fig. 4. It can be easily seen from the figure that the ACI is suppressed by approximately $20 \mathrm{~dB}$. Only the third harmonic can be regenerated and eliminated, and higher harmonics are ignored. Therefore, the suppression effect on ACI is not ideal from the perspective of the entire channel bandwidth.

When the MP parameters are increased to $K=5$ and $Q=9$, the corresponding ACI suppression results are shown in Fig. 5. Since more high-order nonlinear harmonics are suppressed, the residual interference is much smaller than the case of $K=3$ and $Q=3$. It is not difficult to see from the figure that about $40 \mathrm{~dB}$ ACI is suppressed, which can significantly increase the SINR of the desired signal. 
The comparison of the bit error rate (BER) of the system with and without ACI suppression is shown in Fig. 6. The SINR in the figure represents the power ratio of the desired signal to the ACI plus noise in the RX branch. It is not difficult to see from the figure that when the ACI is suppressed, the bit error rate of the system has been significantly improved, which proves the effectiveness of the proposed method.

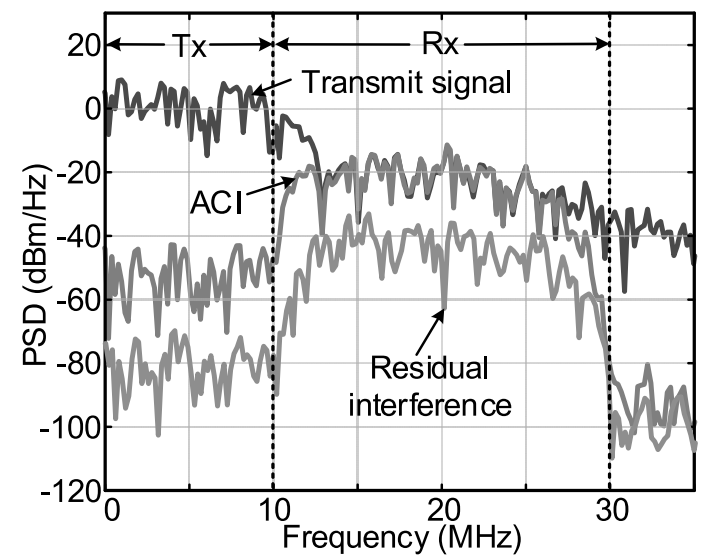

Fig. 4. Simu. result of ACI suppression when $K=3$ and $Q=3$.

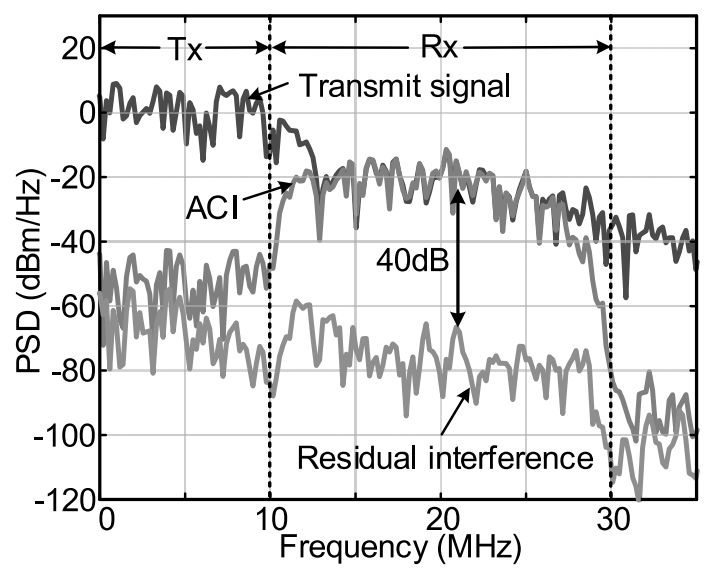

Fig. 5. Simu. result of ACI suppression when $K=5$ and $Q=9$.

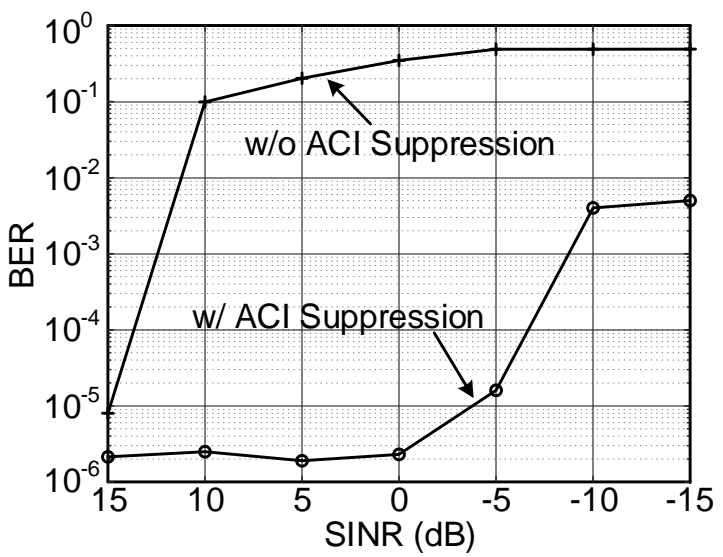

Fig. 6. Comparison of BER with and without ACI suppression.

\section{Experimental Results}

To further test the effectiveness of the proposed method, experimental measurements are conducted on a real PA. The test platform setup includes a PC, a software-defined radio (SDR) platform, attenuators, and a PA, as shown in Fig. 7 [21], [22]. The SDR transmit chain and receive chain are used to realize spontaneous transmission and selfreceiving. The transmit chain includes upconversion circuit and DAC (12J4000 from Texas Instruments). The receive chain includes downconversion circuit and ADC (AD9129 from Analog Devices).

The photo of the experimental platform and the corresponding principle block diagram are shown in Fig. 7.

In the transmit chain, a $20 \mathrm{MHz}$ baseband signal modulated by 16 QAM is first generated, then the baseband signal is upconverted to a carrier frequency at $2.4 \mathrm{GHz}$ and sent to the DAC on the SDR via ethernet. The output analog signal of DAC is fed to the PA (Mini-Circuits ZX60-V82+) and attenuator $(40 \mathrm{~dB})$. To ensure that the PA can generate sufficient nonlinear distortion, multiple active PAs can be connected in series if necessary.

In the receive chain, the signal was downconverted and re-digitized, and then the side lobe of the transmitted signal is taken out with a Matlab band-pass filter (the center frequency is $\omega_{2}$ ) to obtain the ACI. At the same time, an auxiliary branch is constructed in Matlab, and the transmitter baseband signal and the receiver ACI were employed for parameters estimation. Finally, the interference was regenerated and subtracted from the received signal.

For a fair comparison, all the experimental conditions were set the same as that in simulation. The experimental results of the proposed method for suppressing ACI at $K=3$ and $Q=3$ are shown in Fig. 8. The residual interference after suppression is significantly reduced compared against the original ACI. Since the MP model parameter values are set small, the system can only suppress low-order third nonlinear harmonics. It can be seen from the spectrum that the residual interference is relatively large between $10 \mathrm{MHz}-20 \mathrm{MHz}$, which is not conducive to the improvement of the SINR of the desired signal.

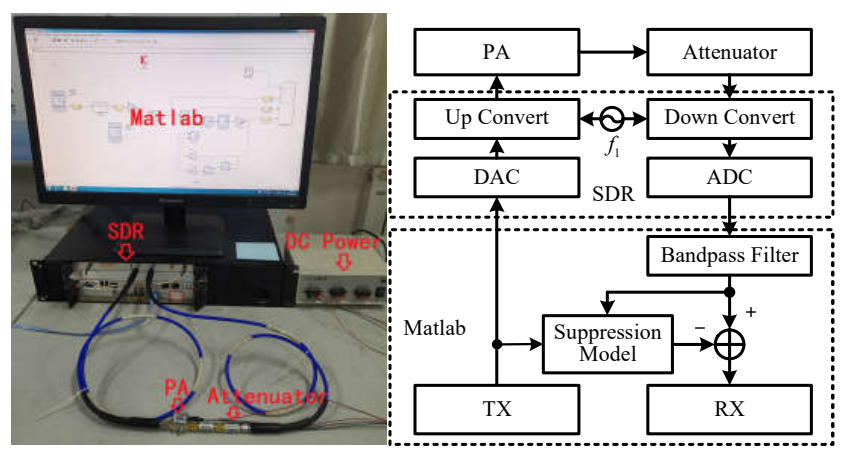

Fig. 7. Laboratorial photo and the corresponding block diagram of the experiment setup. 
The experimental results of the proposed method for suppressing ACI at $K=5$ and $Q=9$ are shown in Fig. 9. As can be seen from the figure, because the system nonlinear characteristics are described in more detail, the MP model can suppress high-order nonlinear distortion. Compared against Fig. 8, the interference suppression effect has been significantly improved. The system can suppress the ACI of about $25 \mathrm{~dB}$, which can effectively improve the SINR of the desired signal.

Table 2 gives a comparison of the experimental results between current approaches and the proposed method.

Compared against the interference suppression results in [10], [14], the proposed method has a substantial improvement in suppressing ACI under the premise of simplifying the circuit structure, which further verifies the effectiveness and feasibility of this method. It also provides necessary data support for the practical application of interference suppression.

\begin{tabular}{|c|c|c|c|}
\hline & Work in [10] & Work in [11] & This work \\
\hline Processing domain & RF & Baseband & Baseband \\
\hline Sample rate & $20 \mathrm{MSPS}$ & $61.44 \mathrm{MSPS}$ & $20 \mathrm{MSPS}$ \\
\hline Circuit complexity & High & Low & Low \\
\hline ACI suppresion & $20 \mathrm{~dB}$ & $21.6 \mathrm{~dB}$ & $25 \mathrm{~dB}$ \\
\hline
\end{tabular}

Tab. 2. The comparation of experimental results.

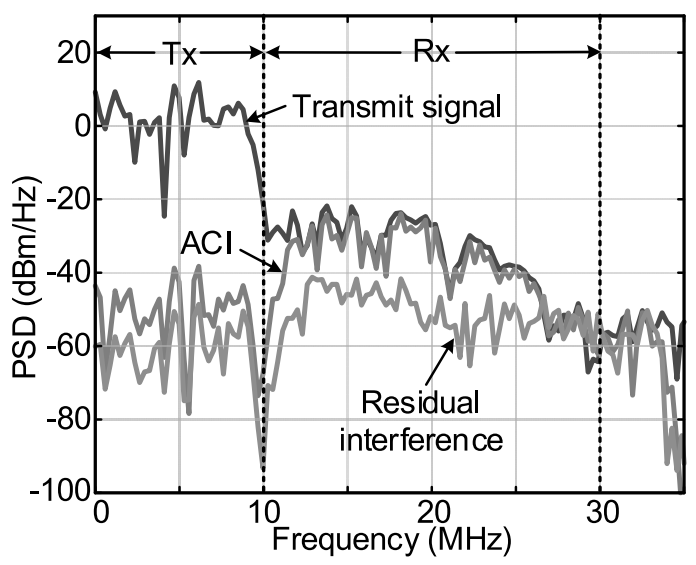

Fig. 8. Exp. result of ACI suppression when $K=3$ and $Q=3$.

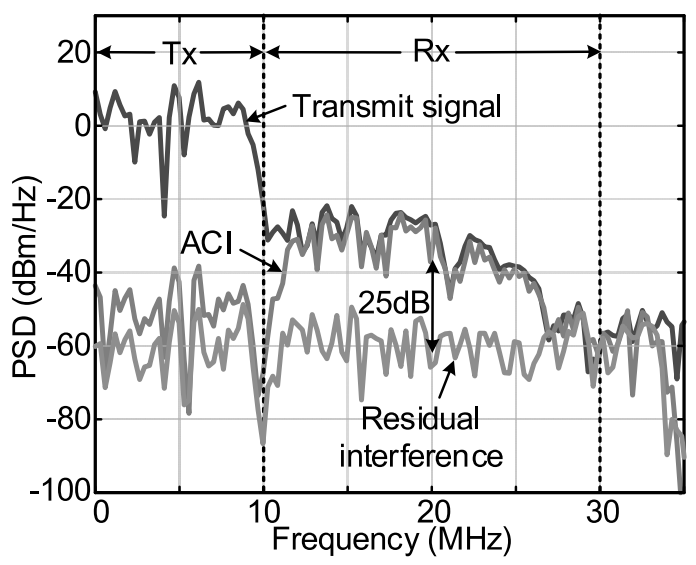

Fig. 9. Exp. result of ACI suppression when $K=5$ and $Q=9$.

\section{Conclusion}

This paper proposed a suppression method to eliminate the ACI in FDD transceiver by using the transmitter leakage and baseband signal. The interference regeneration and cancellation were carried out in the digital baseband domain, which is preferable compared to RF domain complexity. There is also no need for high-rate sampling rate ADC in the proposed method, which is substantially convenient for engineering realization. Computer simulation results and experimental circuit results show that the proposed method can effectively suppress the ACI, thereby improving the SINR of the desired signal. This paper also provided a new method reference for the engineering application of ACI suppression.

\section{Acknowledgments}

This work was supported in part by the National Key R\&D Program of China under Grant 254, in part by the National Natural Science Foundation of China under Grant U19B2014, Grant 61771107, Grant 62071094, Grant 61701075, Grant 61601064, and Grant 61531009, and in part by the Sichuan Science and Technology Program under Grant 2020 YFH0101.

\section{References}

[1] LAUGHLIN, L., ZHANG, C., BEACH, M. A., et al. A 700-950 MHz tunable frequency division duplex transceiver combining passive and active self-interference cancellation. In Proceedings of IEEE MTT-S International Microwave Symposium Digest. Philadelphia (USA), 2018, p. 1-4. DOI: 10.1109/MWSYM.2018.8439253

[2] NIKITIN, A.V., DAVIDCHACK, R.L., SMITH, J.E. Out-of-band and adjacent-channel interference reduction by analog nonlinear filters. EURASIP Journal on Advances in Signal Processing. 2015, vol. 12, p. 1-20. DOI: $10.1186 / \mathrm{s} 13634-015-0202-5$

[3] KESHAVARZ, R., MIYANAGA, Y., YAMAMOTO, M., et al. Metamaterial-inspired quad-band notch filter for LTE band receivers and WPT applications. In Proceedings of General Assembly and Scientific Symposium of the International Union of Radio Science. Rome (Italy), 2020, p. 1-5. DOI: $10.23919 /$ URSIGASS49373.2020.9232331

[4] CALDERIN, L., RAMAKRISHNAN, S., PUGLIELli, A., et al. Analysis and design of integrated active cancellation transceiver for frequency division duplex systems. IEEE Journal of Solid-State Circuits, 2017, vol. 52, no. 8, p. 2038-2054. DOI: $10.1109 /$ JSSC.2017.2700360

[5] KESHAVARZ, R., MOVAHHEDI, M. A compact and wideband coupled-line coupler with high coupling level using shunt periodic stubs. Radioengineering, 2013, vol. 22, no. 1, p. 323-327. ISSN: $1210-2512$

[6] KESHAVARZ, S., ABDIPOUR, A., MOHAMMADI, A., et al. Design and implementation of low loss and compact microstrip triplexer using CSRR loaded coupled lines. AEU - International Journal of Electronics and Communications, 2019, vol. 111, p. 152913-152918. DOI: $10.1016 /$ j.aeue.2019.152913 
[7] FU, Z., ANTTILA, L., ABDELAZIZ, M., et al. Frequency-selective digital predistortion for unwanted emission reduction. IEEE Transactions on Communications, 2015, vol. 63, no. 1, p. 254-267. DOI: 10.1109/TCOMM.2014.2364571

[8] FARSI, S., GHEIDI, H., DABAG, H. T., et al. Modeling of deterministic output emissions of power amplifiers into adjacent receive bands. IEEE Transactions on Microwave Theory and Techniques, 2015, vol. 63, no. 4, p. 1250-1262. DOI: 10.1109/TMTT.2015.2407881

[9] RAMAKRISHNAN, S., CALDERIN, L., NIKNEJAD, A., et al. An FD/FDD transceiver with $\mathrm{RX}$ band thermal, quantization, and phase noise rejection and $>64 \mathrm{~dB}$ TX signal cancellation. In Proceedings of IEEE Symposium on Radio Frequency Integrated Circuits (RFIC). Honolulu (USA), 2017, p. 352-355. DOI: $10.1109 /$ RFIC.2017.7969090

[10] LAUGHLin, L., ZHANG, C., BEACH, M. A., et al. Tunable frequency-division duplex RF front end using electrical balance and active cancellation. IEEE Transactions on Microwave Theory and Techniques, 2018, vol. 66, no. 12, p. 1-13. DOI: 10.1109/TMTT.2018.2851990

[11] ZHANG, C., LAUGHLin, L., BEACH, M. A., et al. A selfinterference cancellation testbed for full-duplex transceiver prototyping. In Proceedings of IEEE International Symposium on Personal, Indoor, and Mobile Radio Communications (PIMRC). Valencia (Spain), 2016, p. 1-6. DOI: 10.1109/PIMRC.2016.7794587

[12] ABDElHaleM, S. H., GUDEM, P. S., LARSON, L. E. Tunable CMOS integrated duplexer with antenna impedance tracking and high isolation in the transmit and receive bands. IEEE Transactions on Microwave Theory and Techniques, 2014, vol. 62, no. 9, p. 2092-2104. DOI: 10.1109/TMTT.2014.2338271

[13] DEYNU, F. K., AKPARI, E. W., AKAMA, C. Low-overhead lowcomplexity carrier phase recovery technique for coherent multiband OFDM-based superchannel systems enabled by optical frequency combs. Journal of Microwaves, Optoelectronics and Electromagnetic Applications, 2021, vol. 20, no. 1, p. 173-194. DOI: 10.1590/2179-10742021v20i1965

[14] CAO, W., LI, Y., ZHU, A. Digital suppression of transmitter leakage in FDD RF transceivers: Aliasing elimination and model selection. IEEE Transactions on Microwave Theory and Techniques, 2018, vol. 66, no. 3, p. 1500-1511. DOI: 10.1109/TMTT.2017.2772789

[15] CAO, W., LI, Y., LUO, G. Q., et al. Digital suppression of transmitter leakage in FDD RF transceivers with an enhanced low-sampling rate behavioral model. IEEE Microwave and Wireless Components Letters, 2018, vol. 28, no. 12, p. 1140-1142. DOI: 10.1109/LMWC.2018.2877257

[16] DING, L., ZHOU, G. T., MORGAN, D. R., et al. A robust digital baseband predistorter constructed using memory polynomials. IEEE Transactions on Communications, 2004, vol. 52, no. 1, p. 159-165. DOI: 10.1109/TCOMM.2003.822188

[17] LIU, Y., QUAN, X., PAN, W., et al. Nonlinear distortion suppression for active analog self-interference cancellers in full duplex wireless communication. In Proceedings of IEEE Global Telecommunications Conference (GLOBECOM). Austin (USA), 2014, p. 948-953. DOI: 10.1109/GLOCOMW.2014.7063555

[18] LIU, Y., ROBLIN, P., QUAN, X., et al. A full-duplex transceiver with two-stage analog cancellations for multipath self-interference. IEEE Transactions on Microwave Theory and Techniques, 2017, vol. 65, no. 12 , p. 5263-5273. DOI: 10.1109/TMTT.2017.2752167

[19] LI, C., ZHAO, H., WU, F., et al. Digital self-interference cancellation with variable fractional delay FIR filter for full-duplex radios. IEEE Communications Letters, 2018, vol. 22, no. 5, p. 1082-1085. DOI: 10.1109/LCOMM.2018.2810270
[20] HUO, X., ZHANG, W., GUO, W., et al. Adjacent channel interference suppression to enhance spectrum sharing for co-located devices. In Proceedings of IEEE International Conference on Communications Workshops (ICC Workshops). Montreal (Canada), 2021, p. 1-6. DOI: 10.1109/ICCWorkshops50388.2021.9473834

[21] PAN, W., XIE, L., XIA, X., et al. A digital predistortion method for fast frequency-hopping systems. In Proceedings of IEEE International Symposium on Signal Processing and Information Technology (ISSPIT). Ajman (United Arab Emirates), 2020, p. 1-3. DOI: $10.1109 /$ ISSPIT47144.2019.9001897

[22] GUO, W., ZHAO, H., TANG, Y. Testbed for cooperative jamming cancellation in physical layer security. IEEE Wireless Communications Letters, 2020, vol. 9, no. 2, p. 240-243. DOI: 10.1109/LWC.2019.2950303

\section{About the Authors...}

Xiaolei HUO was born in Hebei, China, in 1980. He received his B.E. degree and M.E. degrees from Hebei University of Technology in 2002 and 2005 respectively. His research interests include anti-jamming communication and full-duplex wireless communications.

Wenbo GUO was born in Sichuan, China, in 1994. He received the B.E. degree in Automation Engineering and the $\mathrm{Ph} . \mathrm{D}$. degree in Communication and Information Systems from the University of Electronic Science and Technology of China (UESTC), Chengdu, China, in 2015 and 2021, respectively. He is currently a Post-Doctoral Researcher with the National Key Laboratory of Science and Technology on Communications, UESTC. His research interests include interference cancellation, physical layer security, full-duplex communications, and signal processing in wireless communications.

Ying LIU was born in Sichuan, China, in 1985. He received his M.E. and Ph.D. degrees from the University of Electronic Science and Technology of China in 2011 and 2016 respectively. His research interests include digital predistortion, full-duplex wireless communications.

Hongzhi ZHAO was born in Hebei, China, in 1978. He received his $\mathrm{Ph} . \mathrm{D}$. degree from the University of Electronic Science and Technology of China in 2008. His research interests include full-duplex wireless communications, and signal processing in wireless communications.

Youxi TANG was born in Henan, China, in 1963. He received his M.E. and Ph.D. degrees from the University of Electronic Science and Technology of China (UESTC), Chengdu, China, in 1993 and 1997 respectively. His current research interests include spread spectrum system and wireless mobile system with an emphasis on signal processing in communications. 